\title{
Non-alcoholic Steatohepatitis: From Pathophysiology to Clinical Practice
}

\author{
Sherwyn Schwartz, ${ }^{1}$ Jean Lucas ${ }^{2}$ and Mark H DeLegge ${ }^{3}$ \\ 1. Evolution Research Group, San Antonio, TX, USA; 2. Lucas Research, Moorehead City, NC, USA; 3. IQVIA, Raleigh, NC, USA
}

DOI: https://doi.org/10.17925/EE.2021.17.2.112

$\mathrm{N}$ on-alcoholic steatohepatitis (NASH) is becoming a global disease with significant associated comorbidities. To date, there are no commercialized drugs to treat NASH, outside of India; however, there is an abundance of new molecular entities which are in clinical development, some in phase III trials. Many of these trials have created an especially heavy demand for USA-based subjects. Hepatologists currently play a major role in the diagnosis, treatment and clinical-trial enrolment of patients with NASH. However, NASH has a strong metabolic component, with patients often carrying comorbid diseases, such as type 2 diabetes mellitus, obesity, hyperlipidaemia, hypothyroidism and sex steroid disorders. The primary care physician, internist and endocrinologist stand at a pivotal position in the NASH healthcare delivery system, as many of the diseases they commonly encounter are associated with a higher risk of developing NASH. Specialty society practice guidelines are evolving regarding the identification and care of patients with NASH. This review of the literature, and assessment of IQVIA's proprietary patient claims database of diagnosis codes, patient encounters and treatments, substantiates the importance of the primary care provider and endocrinologist in the clinical care and clinical research of patients with NASH.

\section{Keywords}

Non-alcoholic steatohepatitis, metabolic disease, type 2 diabetes mellitus

Disclosure: Sherwyn Schwartz, Jean Lucas and Mark H DeLegge have no financial or non-financial relationships or activities to declare in relation to this article.

Acknowledgements: The authors wish to express their gratitude to and acknowledge the assistance of Bernadette Collins, IQVIA, and Luke Snedacker, Lucas Research, with the collection and preparation of some of the discussed in this article.

Review process: Double-blind peer review.

Compliance with ethics: This study involves a review of the literature and did not involve any studies with human or animal subjects performed by any of the authors.

Authorship: The named authors meet the International Committee of Medical Journal Editors (ICMJE) criteria for authorship of this manuscript, take responsibility for the integrity of the work as a whole, and have given final approval for the version to be published.

Access: This article is freely accessible at

touchENDOCRINOLOGY.com. (C) Touch Medical Media 2021.

Received: 2 June 2020

Accepted: 1 February 2021

Published online: 14 September 2021

Citation: touchREVIEWS in Endocrinology. 2021; 17(2):112-20

Corresponding author: Mark H DeLegge, Global GI Center of Excellence, IQVIA, 4057 Longmarsh Rd, Awendaw, SC, 29429 USA. E: mark.delegge@quintiles.com

Support: No funding was received for

the publication of this article.
Non-alcoholic fatty liver disease (NAFLD) consists of non-alcoholic fatty liver (NAFL) and non-alcoholic steatohepatitis (NASH). It is unclear which specific patients progress from NAFL to NASH and, ultimately, to cirrhosis. However, there is general consensus that, for those patients who do progress to cirrhosis (fibrosis stage 4), there is an increased risk of liver-related complications (liver failure, liver cancer) and cardiovascular disease.1,2

The global prevalence of NAFLD is estimated to be approximately $25 \%$ of the population with an estimate of NASH of between $1.5 \%$ and $6.45 \% .{ }^{3}$ Reports state that the progression of NAFL to NASH occurs in approximately $20-25 \%$ of cases, and that $20 \%$ of those patients will ultimately develop cirrhosis. ${ }^{4}$ Current published data suggest that the incidence of NAFLD is greatest in Hispanics, and lowest in African Americans. ${ }^{5}$ No clear consensus has emerged regarding the factors that promote the progression of patients from NAFLD to NASH. What is clear, is that the development of liver fat content of $>6 \%$ leads to metabolic changes in humans, including insulin resistance and hypertriglyceridaemia. ${ }^{6}$

NASH is a large global disease burden; however, the reality is that the at-risk patient population remains largely undiagnosed. Much of this revolves around limited physician awareness of the disease outside of hepatologists, a relatively small specialty group of physicians. Physician groups that are poised to diagnose and manage these patients are endocrinologists, internists and primary care physicians. These clinicians see many patients with comorbid risk factors associated with $\mathrm{NASH}$.

The IQVIA proprietary P360 database in the USA (as accessed by Bernadette Collins, 20 October 2019), contains 421,000 patient records; when reviewed, using the diagnosis of NASH with the presence of a liver biopsy, it notes that the median age for diagnosis is 56-57 years, and that $62 \%$ of the patients are female. An analysis of comorbidities in the same claims database notes common comorbidities of type 2 diabetes mellitus (T2DM), hypertension, hyperlipidaemia and obesity, amongst other more non-specific symptoms and laboratory tests (Figure 1).

The clinical development space in NASH is very competitive, globally. Recruitment rates for NASH clinical trials have been low, and many trials have been delayed in their completion. The majority of principal investigators, to date, have been hepatologists. There remains tremendous opportunity for endocrinologists, internists and primary care physicians to assume the role of the principal investigator in NASH clinical trials, in addition to the diagnosis and management of these patients.

The objective of this literature review and assessment of IQVIA's proprietary claims-based database of ICD-10 codes, patient encounters and treatments, is to demonstrate how this disease fits squarely into the clinical realm of the endocrinologist, internist and primary care physicians, 
Figure 1: Common comorbidities, non-specific symptoms and laboratory tests associated with non-alcoholic steatohepatitis

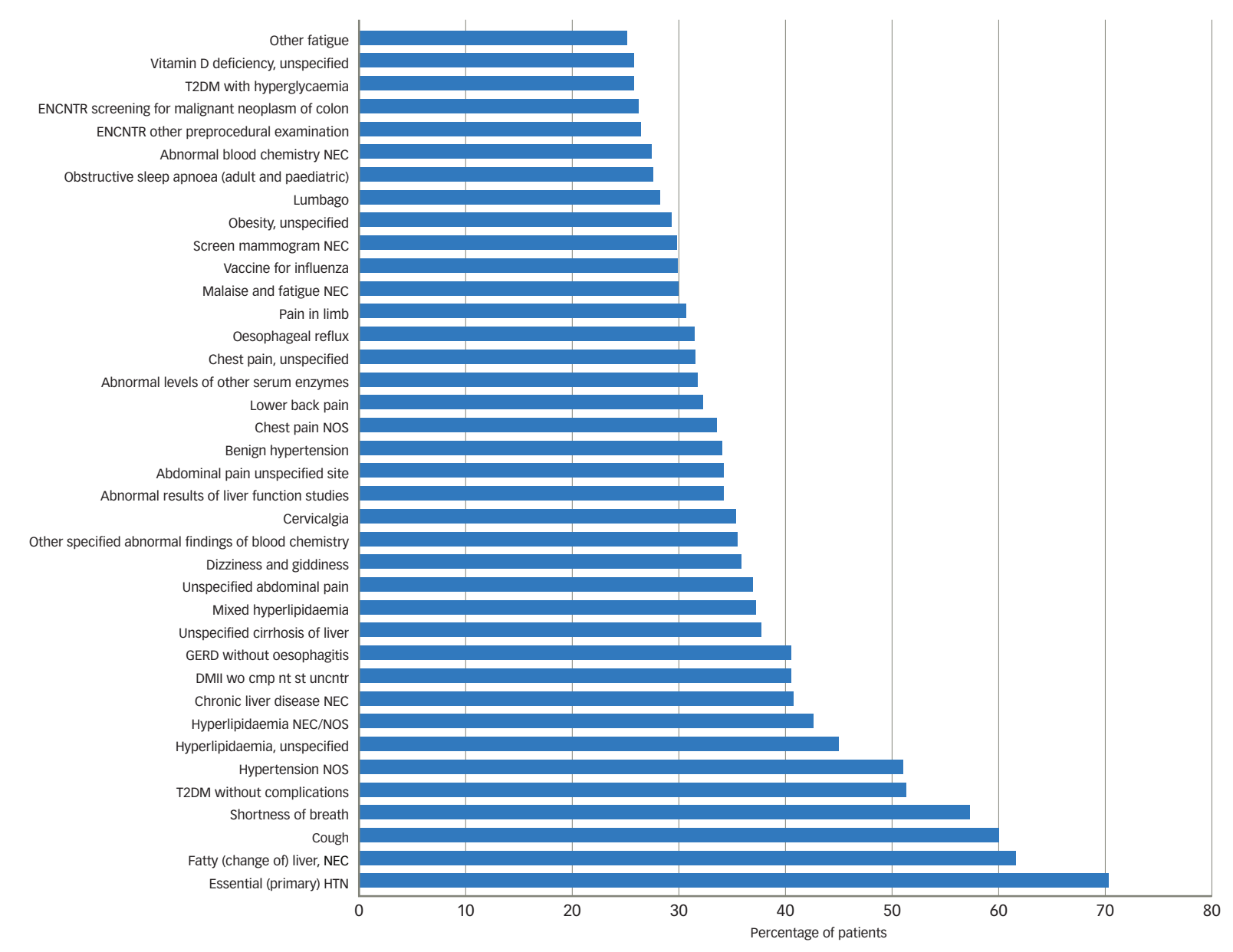

Source: Unadjudicated, unprojected US professional and institutional medical claims processed by Bernadette Collins, IQVIA, accessed 20 October 2019.

DMII wo cmp nt st unchtr= diabetes mellitus without mention of complication, type II or unspecified type, not stated as uncontrolled; ENCNTR = encounter for:

GERD = gastro-oesophageal reflux disease; $H T N$ = hypertension; $N E C=$ not elsewhere classified; NOS = not otherwise classified; T2DM = type 2 diabetes mellitus.

based on disease pathophysiology, current off-label treatments and comorbid disease states. The review also will present current professional endocrinology guidelines related to NAFLD, a proposed patient screening algorithm, suggestions for the operational incorporation of NASH into daily clinical practice, and a review of the opportunities in clinical research for many physician specialties.

\section{Non-alcoholic steatohepatitis pathophysiology}

The exact pathophysiology of NASH remains in question. Some believe that the disease process is a 'two-hit' insult to the liver, while others tout a 'multiple parallel hit' pathway. The two-hit process states that there is an initial accumulation of free fatty acids in the liver, which are derived from visceral adipose tissue.? Visceral adipose tissue accounts for approximately 6-20\% of human adipose tissue; subcutaneous adipose tissue accounts for approximately $80-90 \%$ of whole body-fat tissue. ${ }^{8}$ Within the subcutaneous adipose tissue compartment is a superficial subcutaneous compartment and a deep subcutaneous compartment. The accumulation of fatty acids in the deep subcutaneous compartment also shows a similar positive association with insulin resistance in NASH as the visceral adipose tissue compartment. ${ }^{9}$

The accumulation of free fatty acids leads to insulin resistance. Loss of adipose tissue responsiveness to insulin plays a major role in the pathophysiology of NASH. Failure of insulin to inhibit triglyceride lipolysis in insulin-resistant states leads to an oversupply of free fatty acids to the liver, excess hepatic triglyceride synthesis, and intracellular accumulation of toxic lipid products that impair insulin signalling and activate inflammatory pathways. ${ }^{10}$ This is followed by progression to hepatic inflammation (steatohepatitis), leading to an accumulation of oxygen free radicals, mitochondrial dysfunction and formation of adipokines, all of which result in hepatic tissue damage and fibrosis. Recent data suggest that adipose-based insulin resistance is responsible for the activation of hepatic macrophages and the inflammatory process in the liver. This process is independent of a patient's comorbid disease states of diabetes and/or obesity. ${ }^{11}$

The multiple-hit pathway also includes the free fatty-acid accumulation and inflammation described in the two-hit hypothesis, but believes that the gut produces factors that are important in initiating and advancing hepatic inflammation. The gut-related damage to the liver is derived from cytokine injury and physiologic stress on the hepatic cellular endoplasmic reticulum. ${ }^{12}$ This multiple-hit hypothesis stresses the alterations in the gut microbiome as important in the NASH injury process.

These hypotheses are not all inclusive as to the explanation of NASH pathophysiology. Recent work has confirmed that there is a predisposition to NASH and to significant hepatic fibrosis in people with alterations in the PNPLA3 and TM6SF2 genes. ${ }^{13}$ Although the exact mechanism of this 
increased predisposition and severity is unknown, it is believed to centre around the packaging and export of triglycerides from the hepatocyte. Interestingly, these effects are not related to the presence of insulin resistance or obesity. ${ }^{13}$

The concern with NASH is that it may progress to cirrhosis with the complications of liver failure, including the risk for developing hepatocellular cancer. However, liver dysfunction, failure and cancer are not the leading cause of death for patients with NASH; the leading cause of death is cardiovascular disease. People with NAFLD have a higher risk of developing severe coronary artery disease compared with those who do not have NAFLD. ${ }^{14}$ The presence of fibrosis in patients with NASH is predictive of the occurrence of cardiovascular events. ${ }^{15}$ High calcium scores have been identified in the coronary arteries of patients with NAFLD, independent of metabolic syndrome or obesity. ${ }^{16}$ Furthermore, medical complications associated with T2DM are also higher in patients with NAFLD. In a study of 2,100 patients with T2DM, there were increased rates of chronic kidney disease (odds ratio [OR] 1.87) and retinopathy (OR 1.75) in those with T2DM and NAFLD, compared with those with T2DM without NAFLD. ${ }^{17}$

\section{Non-alcoholic steatohepatitis and the endocrinologist, internist and primary care physician}

Hepatologists commonly diagnose and treat patients with NASH. However, endocrinologists, internists and primary care physicians are specialties that, in general, often see patients with many of the comorbidities associated with NASH (hyperlipidaemia, obesity, T2DM). These physician groups have recently become aware of NASH as a potential common disease entity in their practice setting. Figure 2 depicts which physician specialities are involved in the diagnosis of NASH, based on a large ICD-10 claims database in the USA.

It is becoming increasingly clear that endocrinologists, internists and primary care physicians are making the link between commonly seen disease states and NAFLD, as understanding of NAFLD and its disease history increases. The best-known link is between T2DM and NAFLD. Insulin resistance leads to decreased suppression of gluconeogenesis, a reduction in glycogenesis, and a higher percentage of liver and peripheral tissue fat accumulation. ${ }^{18}$ There is an almost two-fold increased risk of incident T2DM in patients with NAFLD. ${ }^{19}$ In a prospective observational study of patients with T2DM, there was a 69\% prevalence of NAFLD when using ultrasound of the liver to determine the presence of steatosis. ${ }^{20} \mathrm{~A}$ separate systemic review noted the presence of NAFLD in $50-75 \%$ of patients with T2DM. ${ }^{21}$ In people with T2DM, higher glycated haemoglobin (HbA1c) is associated with a higher incidence of hepatic steatosis. ${ }^{22}$ When evaluating the progression to hepatic fibrosis in patients with NASH, T2DM and hypertension are the two most powerful independently associated risk factors. ${ }^{23,24}$ Even in patients without a diagnosis of diabetes, but who demonstrate insulin resistance, there is a higher risk for developing fibrosis of the liver. ${ }^{25}$ Compared with the healthy population, it has been reported that people with T2DM have 2.5-fold increase in the risk of death from cirrhosis. ${ }^{26}$ Chronic liver disease and/or liver cancer are the fourth leading cause of death in patients with T2DM. ${ }^{26}$

\section{Non-alcoholic steatohepatitis and commonly associated co-morbidities}

Obesity is a very common comorbidity in patients with T2DM. Studies have shown that the median prevalence of NASH in patients who are obese is approximately $33 \% .{ }^{27}$ Conversely it has been reported that $51 \%$ of patients with NAFLD and $82 \%$ of patients with NASH are obese. ${ }^{28}$
Figure 2: Treating physicians in patients with non-alcoholic steatohepatitis

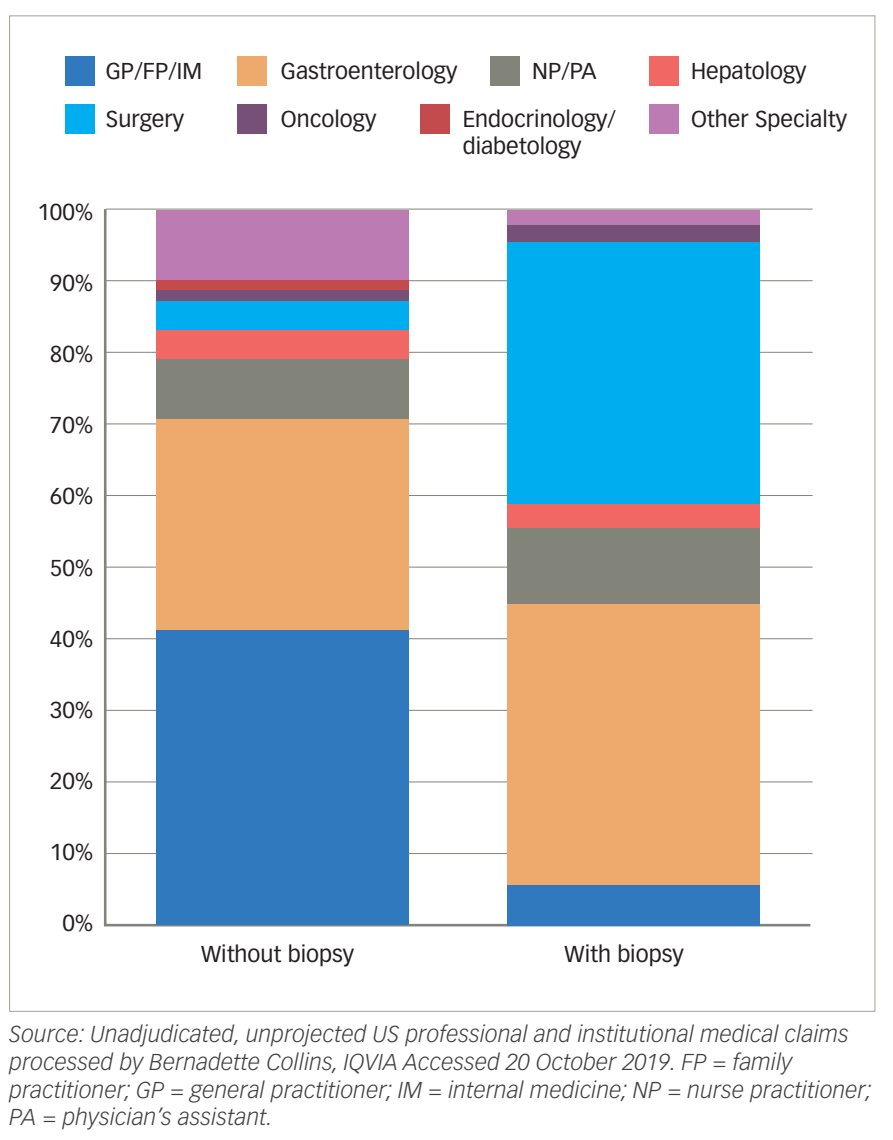

A study from France in 109 patients with NASH noted that gastric bypass surgery and weight loss led to a disappearance of histologic NASH in $85 \%$ of patients..$^{29} \mathrm{~A}$ study from Cuba in patients diagnosed with $\mathrm{NASH}$, evaluated the impact of weight loss by altering the patients' diets. Thirty percent of patients lost $\geq 5 \%$ of their bodyweight. Of those who lost $\geq 5 \%$ bodyweight, a higher proportion experienced NASH resolution and a reduction in disease scores, compared with those who lost $<5 \%$ bodyweight. Patients who lost $\geq 10 \%$ bodyweight were associated with even greater rates of improvement or resolution of steatohepatitis, based on histologically analysed biopsies. ${ }^{30}$

Hyperlipidaemia is associated with NAFLD and is common in patients with T2DM and/or obesity. In the Dallas Heart Study, magnetic resonance spectroscopy was used to measure liver steatosis. By this method, NAFLD was present in $45 \%$ of the Hispanic, $33 \%$ of the Caucasian, and $24 \%$ of the African American patients. ${ }^{31}$

Sex steroids have an impact on the development of NAFLD. Overall, the liver has a major impact on the biological actions of sex steroids. Testosterone and oestrogen, and their derivatives, are metabolized through the liver, which is also where sex hormone binding globulin is produced. The NHANES \| study reported that NAFLD was twice as common in post-menopausal women compared with pre-menopausal women. In post-menopausal women receiving oestrogen treatment, there was a reduced incidence of NAFLD compared with post-menopausal women not receiving oestrogen therapy (OR 0.69)..$^{32}$ In women diagnosed with NASH, the severity of fibrosis was not only associated with being post-menopausal, but also with the number of years a woman had been post-menopausal. ${ }^{33}$ 
Polycystic ovary syndrome (PCOS) is a disease associated with insulin resistance and obesity. ${ }^{34}$ It clinically presents with increased serum androgen levels, amenorrhoea and multiple ovarian cysts. It is believed that $50 \%$ of women with PCOS have insulin resistance..$^{35} \mathrm{~A}$ meta-analysis noted that there is a higher risk of NAFLD in patients with PCOS compared with controls (OR 3.93). From a pathophysiologic standpoint, insulin resistance seems to be the major risk factor for developing NAFLD in PCOS by stimulating the release of androgens. ${ }^{36}$ In a retrospective study of 63,000 women with PCOS compared with 121,000 age and body mass index-matched controls, there was a significant increase in the prevalence of NAFLD in the PCOS diagnosed group (OR 2.23, $p<0.001$ ), even when controlling for additional risk factors, such as the presence of diabetes or pre-diabetes. ${ }^{37}$

Testosterone plays a major role in insulin sensitivity and lipid metabolism..$^{38}$ Low testosterone levels are commonly associated with men who have T2DM and/or are obese. ${ }^{39}$ Furthermore, men with low testosterone have a higher percentage of visceral fat. ${ }^{40}$ In a study of 495 men, those determined to have steatosis by liver ultrasound had lower serum testosterone levels. ${ }^{41}$ In a meta-analysis of almost 14,000 men, serum testosterone was lower in men with NAFLD, compared with men without NAFLD. ${ }^{22}$ In this same study, 5,840 women with higher serum testosterone levels were associated with a higher incidence of fatty liver disease. Studies with hypogonadal males using testosterone replacement therapy have noted a reduction in waist circumference, a reduction in body mass index and an improvement in insulin sensitivity. ${ }^{43}$

Disorders of the pituitary gland also have an association with NAFLD, specifically NASH. Growth hormone deficiency is associated with an increased risk of NAFLD, and is known to cause an increase in visceral fat content and insulin resistance..$^{44}$ In a study of 160 obese patients with liver histology demonstrating NASH and a fibrosis score of $\geq 2$, low growth hormone levels of $<0.45 \mathrm{ng} / \mathrm{mL}$ were reported. ${ }^{45}$ Growth hormone deficiencies are more commonly treated by paediatric endocrinologists. Although the focus of the current review is adult NAFLD, paediatric NAFLD is also becoming a well-recognized disease entity. ${ }^{46}$

The thyroid gland plays an important role in the occurrence of hepatic lipogenesis. The effect is manifested through thyroid hormone receptors. Hypothyroidism decreases the uptake of free fatty acids from triglycerides. ${ }^{47}$ In some studies, hypothyroidism has been associated with NAFLD. ${ }^{48}$ Table 1 is an audit of a large, claims-based patient database evaluating the comorbidities associated with the diagnosis of hypothyroidism. Approximately one-fifth of patients with hypothyroidism are obese, and T2DM and hypertension become more common with advancing age. This underscores the number of comorbid metabolic-associated diseases in the hypothyroid patient population. However, the diagnosis of NAFLD and NASH was low, perhaps demonstrating that these diseases are under-recognized in patients with hypothyroidism.

\section{Currently available treatment for non-alcoholic steatohepatitis}

There are no approved pharmacologic therapies for NAFLD or NASH outside of India, where saroglitazar is approved for the treatment of $\mathrm{NASH}$. Weight loss of $>5 \%$ is associated with an approximately $30 \%$ reduction in liver fat content, ${ }^{49}$ and has also been shown to be associated with improvement or resolution of steatohepatitis. ${ }^{50}$ Other studies have shown that weight loss approaching 10\% can improve the NASH Activity Score (steatosis, inflammation and hepatocyte ballooning) in liver histology, but has no impact on existing liver fibrosis. ${ }^{51}$ The biggest
Table 1: Hypothyroidism and associated comorbidities

\begin{tabular}{|l|l|l|l|l|}
\hline & All ages & $0-17$ & $18-39$ & $40-85$ \\
\hline Hypothyroidism, $\mathrm{n}$ & $8,914,918$ & $<6$ & 985,579 & $7,861,791$ \\
\hline T2DM, \% & 24 & 0 & 10 & 26 \\
\hline Obesity, \% & 18 & 0 & 21 & 17 \\
\hline Hypertriglyceridaemia, \% & 11 & 0 & 7 & 18 \\
\hline Hypertension, \% & 51 & 0 & 14 & 56 \\
\hline NAFLD, \% & 2 & 0 & 2 & 2 \\
\hline NASH, \% & 0 & 0 & 0 & 0 \\
\hline
\end{tabular}

Source: Unadjudicated, unprojected US professional and institutional medical claims processed by IQVIA, 2019. Updated 30 September 2019. Accessed

15 October 2019 via IQVIA's P360 query engine by Bernadette Collins, IQVIA. $N A F L D=$ non-alcoholic fatty liver disease; $N A S H=$ non-alcoholic steatohepatitis; T2DM = type 2 diabetes mellitus.

challenge with dietary therapy in clinical practice is the ability of patients to comply with dietary changes over a prolonged period of time.

It would be intuitive for clinicians to consider prescribing currently commercialized medications for the treatment of T2DM, obesity and or hypertriglyceridemia as a potential treatment for NASH. Potential T2DM medications that may be considered as candidates to treat NASH would include metformin, thiazolidinediones, glucagon-like peptide 1 (GLP-1) receptor agonists, dipeptidyl peptidase 4 (DPP-4) inhibitors and sodium-glucose co-transporter 2 (SGLT2) inhibitors. However, the data supporting the use of currently commercialized medications are limited.

To date, studies with metformin, DPP-4 inhibitors and SGLT2 inhibitors have shown no hepatic histologic improvement in patients with NAFLD. ${ }^{52-54}$ In the drug category of thiazolidinediones, there are a few prospective, randomized trials that have been published. A study with rosiglitazone in patients with biopsy-proven NASH demonstrated an improvement in hepatic steatosis but none of the other histologic features of NASH. ${ }^{55} \mathrm{~A}$ meta-analysis of randomized clinical trials using pioglitazone showed a benefit for patients with advanced NASH (F3, F4 fibrosis). ${ }^{56}$ Pioglitazone has been shown to reduce cardiovascular risk in patients with metabolic syndrome and T2DM. ${ }^{57}$ There is potential risk, however, as pioglitazone is associated with peripheral oedema, and may worsen heart failure. The American Association for the Study of Liver Disease (AASLD) notes pioglitazone may be a viable option for the treatment of $\mathrm{NASH}$, but only after the risks and benefits of treatment are explained to the patient. ${ }^{58}$

GLP-1 inhibitors have been shown to reduce liver enzymes (aminotransferases) and steatosis. ${ }^{59}$ In a phase II clinical study of patients with NASH treated with liraglutide for 48 weeks, there were significant reductions of hepatic steatosis, hepatic cell ballooning and liver enzymes compared with patients who received placebo. ${ }^{60} \mathrm{~A}$ meta-analysis of six randomized trials in 4,442 patients with diabetes and NAFLD treated with liraglutide noted an improvement alanine aminotransferase (ALT) and a trend towards improvement in steatosis versus placebo, but no significant changes in inflammation and fibrosis in patients with available liver histology. ${ }^{61}$ Saroglitazar, a peroxisome proliferator-activated receptor agonist, has been approved in India for the treatment of NASH and for the treatment of dyslipidaemia in patients with diabetes. ${ }^{62}$

No improvement in liver histology has been reported with the use of statins compared with placebo in a randomized clinical trial. ${ }^{63}$ Oxidative stress, and more specifically, lipid peroxidation, is believed to play a large role on the pathophysiology of NASH. Antioxidant therapy has been considered 
as a potential treatment.A meta-analysis of clinical trials evaluating various antioxidant interventions, such as vitamins $\mathrm{C}$ and $\mathrm{E}$, showed no benefit of these interventions in patients with NAFLD. ${ }^{64}$ The AASLD suggests that vitamin E may be used in patients with NASH who do not have diabetes. ${ }^{58}$

Obesity surgery has been reported as a viable option for the treatment of NASH in appropriately selected patients. In a retrospective analysis of 64 patients with biopsy-confirmed NASH who received bariatric surgery, $84 \%$ of patients revealed resolution of steatohepatits and $72 \%$ experienced fibrosis regression at follow-up liver biopsy 5 years later. ${ }^{65}$ Bariatric surgery has also been shown to reduce overall cardiovascular mortality in obese patients, compared with usual care. ${ }^{66}$

To date, four global phase III trials have been completed, or are in process, for the treatment of NASH (ClinicalTrials.gov Identifiers: NCT02548351, NCT03053063, NCT03053050, NCT02704403). ${ }^{67-70}$ These drug trials were on an accelerated pathway for approval based on liver histologic outcomes, with the caveat that any drug approved on this pathway for the USA and Europe will require an additional trial to evaluate long-term outcomes (liver outcomes, such as cancer, hepatic failure, liver transplant) and all-cause mortality. Of the completed phase III trials, selonsertib failed its primary outcome for both F3 and F4 fibrosis (ClinicalTrials.gov Identifiers: NCT03053063, NCT03053050), elafibranor failed its primary outcome (ClinicalTrials.gov Identifier: NCT02704403), and obeticholic acid met one of its two primary outcomes (ClinicalTrials.gov Identifier: NCT02548351); however, the FDA did not approve this medication for the treatment of NASH due to a variety of concerns. ${ }^{67-70}$ Resmetirom remains in a phase III trial.

\section{Screening for non-alcoholic fatty liver disease}

In the practice setting, screening patients at high-risk for NASH is an important process. Ultimately, the diagnosis can currently only be achieved by liver biopsy. Despite the fact that there is no currently approved pharmacologic treatment for NASH outside of India, there is still an opportunity for patient education and awareness of available clinical trials.

Some clinicians use elevated liver function tests (LFTS) as a method of screening for patients at risk for NASH. Published data report a rise in the number of LFTs performed in primary care physician practices. ${ }^{11}$ Elevated LFT can be associated with numerous potential diagnoses. In a clinical research setting of 1,118 patients with documented LFT abnormalities, the aetiology of the abnormal LFT was identified in 55\% (614) of these patients. Interestingly, $24.3 \%$ of this group was diagnosed with NAFLD because there was evidence of steatosis on ultrasound examination of the liver, and there were no other identified possible causes for the elevated LFT. ${ }^{72}$ Another research group in a similar setting aimed to determine the degree of fibrosis in their patients with NAFLD, as this would be consistent with a high risk of NASH diagnosis. In this study, the NAFLD Fibrosis Score was used to determine the severity of fibrosis. Of the subjects with NAFLD, 7.6\% had advanced fibrosis, 35.2\% may have had fibrosis and $57.2 \%$ had no fibrosis. ${ }^{73}$ NAFLD is recognized globally as the most common reason a patient presents to their physician with an elevated LFT. ${ }^{74}$

Ultimately, it would be ideal to have a validated screening algorithm that could be used to identify patients at high risk for NASH. Figure 3 is a proposed algorithm for screening for patients with NAFLD who are at high risk of having NASH, and who should have a confirmatory liver biopsy. This algorithm was conceived after interrogation of a large USA claims and treatment database (IQVIA P360 database), in conjunction
Figure 3: Screening algorithm for non-alcoholic steatohepatitis

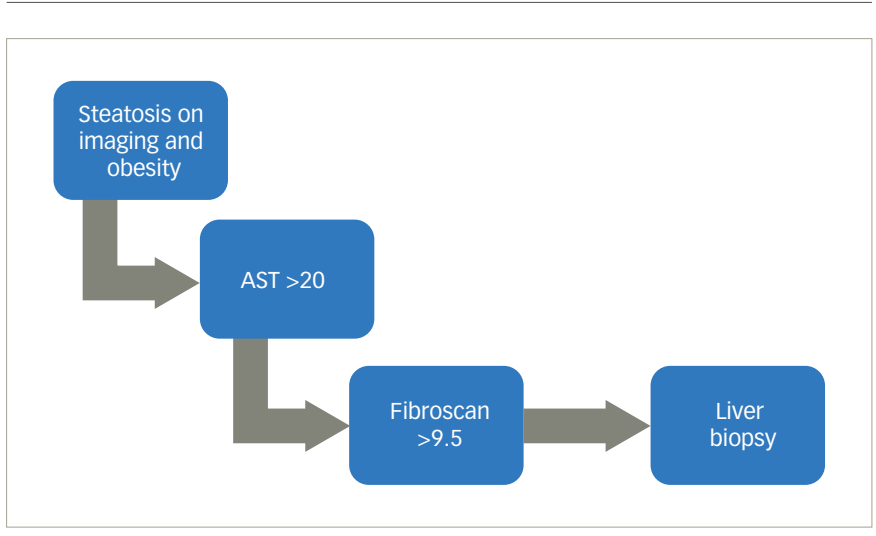

AST = aspartate aminotransferase

with a separate interrogation of an electronic medical record (EMR) of an endocrinology practice in the USA containing patients with both biopsy-positive and biopsy-negative NASH (Allscripts Professional EMR version 17.3.1, as accessed by Luke Snedacker, Lucas Research, November 2019).

There are peer-reviewed publications which have reviewed the use of non-invasive biomarkers for the diagnosis of NAFLD. ${ }^{75}$ There are a number of commercialized assays for detecting NAFLD with varying sensitivity and specificity. These tests have not gained much popularity due to cost and the inability to confirm the diagnosis of NASH. A more economical method is scoring systems, which are based on commonly available laboratory and demographic information in the patient's chart. These scoring systems (e.g. Fib-4 and NASH Fibrosis Score) are focused on determining whether a patient has liver fibrosis, a pathological finding in NASH. These scoring systems are better able to determine if a patient has no fibrosis or severe fibrosis, compared with mild or moderate fibrosis. A bedside medical device is available for measuring the degree of liver stiffness (fibrosis) or liver fat content; this is known as transient elastography. This device has good sensitivity and specificity for determining the absence or presence of extensive liver fat or fibrosis. It does not allow accurate specific fibrosis or steatosis grading and cannot substitute for liver biopsy for the diagnosis of NASH.

\section{Endocrine/diabetes societies and non-alcoholic fatty liver disease}

Globally, endocrine societies are developing recommendations for identifying patients with NASH in the endocrinologist's practice. The Chinese Society of Endocrinology recently published guidelines which advocate ultrasound for the screening of patients at high risk for NAFLD, and the use of the NASH Fibrosis Score to determine which patients should be sent for liver biopsy. ${ }^{76}$ The increased prevalence of NAFLD prompted the American Diabetes Association to revise its 2019 recommendations for standard of medical care. In adults, adolescents and younger children, the recommendations are to conduct liver enzyme (aspartate aminotransferase [AST] and ALT) testing at the initial visit, and annually thereafter. ${ }^{77}$ In 2016, the European Association for the Study of the Liver, the European Association for the Study of Diabetes and the European Association for the Study of Obesity (EASO) published clinical practice guidelines for the management of NAFLD. Box 1 summarizes the major recommendations. ${ }^{78}$

Referral to gastroenterologist/hepatologist is recommended for persistently elevated or worsening transaminases in all age groups. 
Box 1: EASL-EASD-EASO major summary recommendations for the clinical management of non-alcoholic fatty liver disease

- Patients with insulin resistance and obesity or metabolic syndrome should undergo testing to diagnose excess liver fat

- Patients with liver steatosis should have a careful assessment of daily alcohol intake

- When diagnosing NAFLD, other causes of liver disease should also be considered

- Patients with liver steatosis should be screened for metabolic syndrome

- Patients with elevated liver function test should be screened for NAFLD

- In patients with metabolic syndrome, screening for NAFLD should include an evaluation of liver enzymes and liver ultrasound

- Specific genotyping for high-risk patients with NAFLD is not recommended

- Liver ultrasound is the preferred screening imaging for NAFLD

- Quantitative liver fat content is best obtained by MRI spectroscopy

- NASH must be diagnosed by a liver biopsy

- Transient elastography and scoring systems are acceptable non-invasive biomarkers for identifying low-risk liver fibrosis patients (not cirrhosis)

- Advanced liver fibrosis (cirrhosis) must be confirmed by a liver biopsy

- Patients with NAFLD should be screened for diabetes

- Patients with T2DM should be screened for NAFLD, irrespective of serum liver enzyme levels

- Patients with NASH or fibrosis and hypertension should be closely monitored for progression of fibrosis

- Patients diagnosed with NASH should be screened for cardiovascular disease

- Patients with NASH or fibrosis should be treated with dietary and exercise counselling. Pharmacologic treatment cannot be recommended. Pioglitazone or vitamin E can be considered

- Diabetes control is recommended for patients with NASH or fibrosis

$E A S D=$ European Association for the Study of Diabetes; EASL = European Association for the Study of the Liver; EASO = European Association for the Study of Obesity;

$M R I=$ magnetic resonance imaging: NAFLD = non-alcoholic fatty liver disease:

$\mathrm{NASH}=$ non-alcoholic steatohepatitis; $T 2 D M=$ type 2 diabetes mellitus.

These elevated enzymes should be closely monitored in patients with higher body mass index, higher waist circumference, elevated triglyceride levels and lower high-density lipoprotein cholesterol levels. Even before referral to gastroenterology/hepatology, the endocrinologist should maximize glycaemic, lipid and weight-loss therapies. In appropriate patients, glycaemic therapy should consider treatment with liraglutide or thiazolidinediones as an off-label treatment for NAFLD, as they have been shown to have some promise in preliminary studies. ${ }^{79}$ Published data in a broad population of patients at high risk of NAFLD have demonstrated that $72 \%$ of patients are either counselled on weight loss, or attempt weight loss on their own. Twenty percent of these patients were able to obtain a weight loss of $5 \%$ or greater at 1 year. ${ }^{80}$

As discussed previously, the aetiology of NASH is thought to stem from visceral adiposity and insulin resistance. Morbidity in this disease is related to both liver decompensation and heart disease. This points to NASH being a systemic disease. A study by Targher et al. evaluated three groups of patients, each including 45 males who were either healthy, overweight with biopsy-proven NASH, or overweight without ultrasound diagnosed hepatic steatosis. Patients with NASH had the highest levels of C-reactive protein, PAl-1 activity, triglycerides and HOMA level (indicator of insulin resistance), and the lowest adiponectin level..$^{81}$ Patients with $\mathrm{NASH}$ in endocrinology practices who are the most insulin resistant, also have the worst cardiac risk factors and the highest ALT levels. The association between insulin resistance and obesity explains why these patients with liver disease have a high degree of cardiac disease
Before referral to the gastroenterologist/hepatologist for elevated or worsening levels of liver enzymes, simple scoring algorithms, such as the Fib-4 and AST to Platelet Ratio Index (APRI), can be used to predict the presence of hepatic fibrosis. A Fib- 4 score of $<1.45$ has a $90 \%$ negative predictive value for advanced fibrosis, whereas a score $>3.25$ would have a $97 \%$ specificity and a positive predictive value of $65 \%$ for advanced fibrosis. ${ }^{82}$ For APRI, scores $>0.7$ have a sensitivity of $77 \%$ and specificity of $72 \%$ for predicting significant hepatic fibrosis. ${ }^{83}$

Because of the rising incidence of diabetes and obesity, NASH-related disease morbidity will be almost as common as the cardiac problems seen in many routine clinical practices. Therefore, it will be important in the non-gastroenterology practice to become versed in the diagnosis and care of patients with NASH. In the future, as with cardiac disease and referrals to cardiologists, referrals to hepatologists for NASH will occur when disease presentation is unusual or the patient needs an invasive procedure.

Non-invasive biomarkers will be critical for the awareness and diagnosis of NAFLD by endocrinologists. At present, research is looking at promising tests, such as plasma cytokeratin 18, oxidized low-density lipoprotein, interleukin-17 and others. ${ }^{84}$ Most endocrine, internal medicine and primary care practices will need to have access to a transient elastography device, which will give an estimate of hepatic fat and fibrosis presence at the bedside. This device, in combination with predictive algorithms or serum or imaging biomarkers, will assist these physician specialties in determining which patients are at risk for NAFLD, and which patients will require a liver biopsy for diagnosis and staging of disease.

\section{Clinical research in non-alcoholic steatohepatitis}

Patients with risk factors for NASH should be followed closely with management of associated metabolic abnormalities, and should receive a referral to a dietician and education regarding exercise. In a meta-analysis of clinical trials evaluating the efficacy of exercise in NAFLD, moderate-intensity exercise demonstrated a beneficial effect on liver fat content, independent of its impact on body weight. ${ }^{85}$ Screening for NAFLD should be part of the routine care for these patients in order to identify and educate patients with a diagnosis of NASH. In a population-based study in Europe, one in six patients with T2DM were non-invasively determined to have liver fibrosis using a combination of liver ultrasound and transient elastography. ${ }^{86}$ Once a diagnosis of NASH has been made, consideration for enrolment into clinical trials should also be considered.

Over the past 10 years, the number of clinical trials focusing on patients with NASH has increased dramatically. The current regulatory pathway allows approval of a drug to treat NASH based on very specific histologic improvement over a 52-72-week interval. However, longer-term data from liver outcome studies (carcinoma, liver failure, transplant) and all-cause mortality data will need to be collected over a longer period of time (3-5 years) to allow a drug to maintain its approved status, and to demonstrate true clinical efficacy outcomes to payers, regulatory agencies and clinicians. Patients entering a phase III trial may need to remain in that clinical study for a prolonged period of time. Between the increasing number of clinical trials and the length of time of phase III trials, there will be a continued high demand for subjects to enrol in these trials.

Currently, there are 52 NAFLD pharmaceutical trials underway globally. Figure 4 demonstrates the current status of pharmaceutical trials 


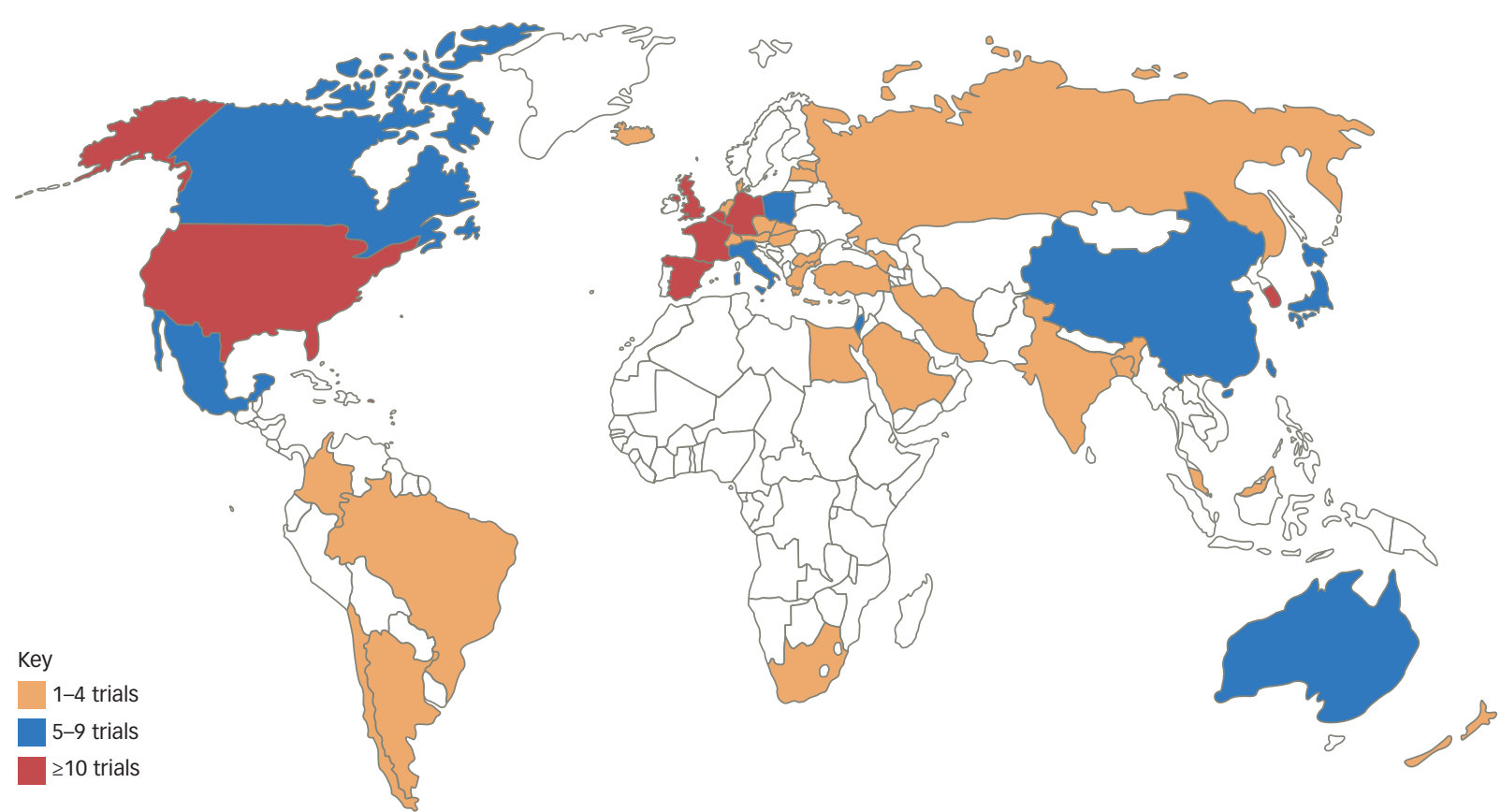

Figure data as of March 2021. Fifty-two trials currently open to enrolment (phases I-IV) targeting approximately 11,332 patients with non-alcoholic steatohepatitis Source: Trialtrove ${ }^{\circledR}$ Informa, 2021, as accessed by Nicole King PhD IQVIA on 26 March 2021.

being performed in NAFLD as of 26 March 2021. Based on the data captured in Figure 4, the current volume of NAFLD studies will require 12,085 patients to fulfil their patient enrolment requirements.

\section{Introduction of non-alcoholic steatohepatitis into clinical practice}

Incorporation of the identification and care of patients with NASH into a clinical practice will be a shift for many clinicians, irrespective of their location at an academic medical centre or in the community. There is already a multitude of care issues in specific patient groups, such as those with T2DM or hyperlipidaemia, that must be addressed by physicians. This includes monitoring for the development of associated complications by providing routine cardiac evaluation, renal function assessment and ophthalmologic care. A focus on weight loss and control of $\mathrm{HbA} 1 \mathrm{C}$ and plasma lipids is already the standard of care. Attention to the liver and the development of NASH will now need to be considered an important end-organ complication for many of the patients commonly seen in the endocrinology, internal medicine or primary care practice.

The ability to identify patients at risk for $\mathrm{NASH}$, and to decide who may need to be referred to a hepatologist, will be an important component of each clinical practice's standard of care. There is no one serum blood test or a radiograph that can identify a patient with NASH. However, physicians can stratify patients for further evaluation based on several factors including:

- treatment of patients with multiple metabolic-related disease states including obesity, T2DM, hypertension, hyperlipidaemia, hypothyroidism or PCOS

- elevated abdominal waist circumference

- platelet count $<200,000$

- abnormal LFT

- steatosis determined on imaging (ultrasound, computerized tomography or magnetic resonance imaging).
These patients should have an assessment of their hepatic fibrosis risk using an algorithm, such as the Fib-4 score or NASH Fibrosis Score. In some practices, the use of bedside transient elastography can be employed for further determination of hepatic steatosis and fibrosis. The current device for performing transient elastography is expensive, and reimbursement for performing the procedure is low. Some practices may elect to refer patients for transient elastography to a hepatologist, or to defray the cost of the transient elastography device by enrolling patients in NASH clinical research trials, where transient elastography has proven useful in the screening process for identifying patients who may be candidates for a given trial.

In the USA, liver biopsies are now often performed by interventional radiologists, and the tissue sample is read by a pathologist. A clinician faced with the possible diagnosis of NASH in a patient with presumptive liver fibrosis, especially moderate to severe fibrosis, may elect to send them for liver biopsy, especially given the fact that there is currently no commercialized pharmaceutical treatment option. Alternatively, they may elect to refer these patients to a hepatologist to make this decision. In either case, the focus is on the patient, the diagnosis of NASH, and the current treatment options, which include aggressive weight loss, control of hypertension and hyperlipidaemia, education on alcohol intake, and optimization of blood sugar control. Additionally, these patients can be considered for inclusion into a clinical trial.

Using claims data from a large USA database, we have presented a snapshot of the comorbidities, non-specific symptoms and laboratory tests associated with NASH (Figure 1). A further evaluation of a community-based endocrinology practice electronic medical records of 122 biopsied patients with NASH revealed associated comorbidities, as seen in Table 2. Comparing the claims-based data with a community-based endocrinology practice electronic medical records revealed the same female predominance of the disease. Hypertension, 
Table 2: Comorbid disease processes as identified by office-based electronic medical record $(\mathrm{N}=122)$

\begin{tabular}{|c|c|c|c|}
\hline Females (65\%) & Percent (of N) & Males (35\%) & Percent (of N) \\
\hline NASH & 45 & $\mathrm{NASH}$ & 21 \\
\hline Fatty liver & 42 & Hypertension & 20 \\
\hline Hypertension & 41 & Fatty changes of the liver & 16 \\
\hline Vitamin D deficiency & 36 & T2DM & 15 \\
\hline T2DM & 33 & Hyperlipidaemia & 13 \\
\hline Hyperlipidaemia & 25 & T2DM With hyperlipidaemia & 12 \\
\hline Major depressive disorder & 23 & Gastro-oesophageal reflux disease & 11 \\
\hline Gastro-oesophageal reflux disease & 23 & Hypercholesterolaemia & 9 \\
\hline Constipation & 21 & Polyneuropathy & 8 \\
\hline Osteoarthritis & 21 & Fatigue & 8 \\
\hline
\end{tabular}

Source: Allscripts Professional EMR version 17.3.1, accessed by Luke Snedacker, Lucas Research, November 2019. Database represents patient records from one US-based endocrinology practice.

$\mathrm{NASH}=$ non-alcoholic steatohepatitis; $T 2 D M=$ type 2 diabetes mellitus.

T2DM and hyperlipidaemia were common comorbidities, and were numerically more common in females. In the office-based EMR analysis, fatty liver disease was a common comorbidity. Surprisingly, obesity was not a common comorbidity in the office-based EMR analysis; this could be the result of the office coding practice, where the presence of obesity is often under-recognized and under coded.

\section{Conclusion}

NAFLD, and specifically NASH, is becoming a growing healthcare concern. A recent healthcare economic model forecasted that NASH has become the fastest-growing category of liver disease requiring transplant over the past 20 years.87 The endocrinologist, internist and primary care physician stand at a pivotal position in the healthcare delivery system, as many of the diseases they commonly treat are associated with a higher risk of developing NASH. Currently there is only one drug approved for NASH (saroglitazar), and this is only approved in India. Pioglitazone and vitamin E are being used off-label for the treatment of NASH by some clinicians. Practice guidelines for endocrinologists are evolving by bringing into focus the identification and care of patients with NASH in their daily clinical practice. The endocrinologist, internist and primary care physician will play an increasingly important role in the clinical care and clinical research of the NASH patient population. $]$
1. Yatsuji S, Hashimoto E, Tobari M, et al. Clinical features and outcomes due to non cirrhosis caused by hepatitis C. Hepatology. 2008;24:248-54. Bertot LC, Adams LA. The natural history of non-alcoholic fatty liver disease. Int J Mol SCi. 2016;29:774-85.

3. Younassi ZM. The epidemiology of nonalcoholic steatohepatitis. Clin Liv Dis. 2018;11:92-4

4. Angulo P, Alba LM, Petrovic LM, et al. Leptin, insulin resistance, and liver fibrosis in human nonalcoholic fatty liver disease. J Hepatol. 2004;41:943-9.

5. Williams CD, Stengel J, Asike MI, et al. Prevalence of nonalcoholic fatty liver disease and nonalcoholic steatohepatitis among a largely middle-aged population utilizing ultrasound and liver biopsy: a prospective study. utilizing ultrasound and liver biopsy:

6. Bril F, Barb D, Portillo-Sanchez $P$, et al. Metabolic and histological implications of intrahepatic triglyceride content in nonalcoholic fatty liver disease. Hepatology. 2017;65:1132-44.

7. Masarone M, Federico A, Abenavoli L, et al. Non-alcoholic fatty liver: epidemiology and natural history. Rev Recent Clin Trials. 2014;9:126-33.

8. Smith SR, Lovejou LC, Greenway F, et al. Contributions of total body fat, abdominal subcutaneous adipose tissue compartments and visceral adipose tissue to the metabolic complications of obesity. Metabolism. 2001;50:425-35.

9. Kelley DE, Thaete FL, Troost F, et al. Subdivisions of subcutaneous adipose tissue and insulin resistance. Am J Physiol Endocrinol Metab. 2000;278:E941-8.

10. Lomonaco R, Ortiz-Lopez C, Orsak B, et al. Effect of adipose tissue insulin resistance on metabolic parameters and liver histology in obese patients with nonalcoholic fatty liver disease. histology in obese patients with

11. Rosso C, Kazankov K, Younes R, et al. Crosstalk between adipose tissue insulin resistance and liver macrophages in non-alcoholic fatty liver disease. J Hepatol. 2019;71:1012-21.

12. Tilg H, Moschen AR. Evolution of inflammation in nonalcoholic fatty liver disease: the multiple parallel hits hypothesis. Hepatology. 2010;52:1836-46.

13. Koo BK, Joo SK, Kim D, et al. Additive effects of PNPLA3 and TM6SF2 on the histologic severity of non-alcoholic fatty live disease. J Gastroenterol Hepatol. 2017;33:1277-85.

14. Ekstedt $M$, Hagström $H$, Nasr $P$, et al. Fibrosis stage is the strongest predictor for disease specific mortality in NAFLD after up to 33 years of follow-up. Hepatology. 2015;61:1547-54.

15. Perazzo $H$, Munteanu M, Ngo $Y$, et al. Prognostic value of liver fibrosis and steatosis biomarkers in type-2 diabetes and dyslipidaemia. Aliment Pharmacol Ther. 2014;40:1081-93.

16. Al Rifai M, Silverman MG, Nasir K, et al. Association of nonalcoholic fatty liver disease, obesity, and metabolic syndrome, with systemic inflammation and subclinical atherosclerosis: The Multi-Ethnic Study of Atherosclerosis (MESA). Atherosclerosis. 2015:239:629-33.

17. Targher $G$, Bertolini L, Rodella S, et al. Non-alcoholic fatty liver disease is independently associated with an increased prevalence of chronic kidney disease and proliferative/lase treated retinopathy in type 2 diabetic patients. Diabetologia. 2008;51:444-50.

8. Tilg H, Moschen AR, Roden M. NAFLD and diabetes mellitus. Nat Rev Gastroenterol Hepatol. 2017;14:32-42.

19. Ballestri S, Zona S, Targher G, et al. Nonalcoholic fatty liver disease is associated with an almost twofold increased risk of incident type 2 diabetes and metabolic syndrome: evidence from a systematic review and meta-analysis. I Gastroenterol Hepatol. 2016;31:936-44.

20. Leite NC, Salles GF, Araujo AL, et al. Prevalence and associated factors of non-alcoholic fatty liver disease in patients with type-2 diabetes mellitus. Liver Int 2009:29:113-9.

21. Lonardo A, Ballestri S, Marchesini $G$, et al. Nonalcoholic fatty liver disease: a precursor of the metabolic syndrome. Dig Liver Dis. 2015;47:181-90

22. Portillo-Sanchez P, Bril F, Maximos M, et al. High prevalence of nonalcoholic fatty liver disease in patients with type 2 diabetes mellitus and normal plasma aminotransferase levels. J Clin Endocrinol Metab. 2015;100:2231-8.

23. Singh $S$, Allen AM, Wang $Z$, et al. Fibrosis progression in nonalcoholic fatty liver vs nonalcoholic steatohepatitis: systematic review and meta-analysis of paired-biopsy studies. Clin Gastroenterol Hepatol. 2015;13:643-54.

24. MCPherson S, Hardy T, Henderson E, et al. Evidence of NAFLD progression from steatosis to fibrosing-steatohepatitis progression from steatosis to fibrosing-steatohepatitis
using paired biopsies: implications for prognosis and clinical using paired biopsies: Implications for prog
management. J Hepatol. 2015;62:1148-55.

25. Vidal J, Ferrer JP, Estimates $E$, et al. Diabetes mellitus in patients with liver cirrhosis. Diabetes Res Clin Pract. 1994;25:19-25.

26. de Marco, R, Locatelli F, Zoppini G, et al. Cause-specific mortality in type 2 diabetes. The Verona Diabetes Study. Diabetes Care. 1999;22:756-61.

27. Abrams GA, Kunde SS, Lazenby AJ, Clements RH. Portal fibrosis and hepatic steatosis in morbidly obese subjects: a spectrum of nonalcoholic fatty liver disease. Hepatology. 2004:40:475-83.

28. Younossi ZM, Koenig AB, Abdelatif $D$, et al. Global epidemiology of nonalcoholic fatty liver disease-meta-analytic assessment of prevalence, incidence, and outcomes. Hepatology. 2016;64:73-84.

29. Lassailly $G$, Caiazzo R, Buob D, et al. Bariatric surgery reduces features of nonalcoholic steatohepatitis in morbidly obese features of nonalcoholic steatohepatitis in morbic

30. Vilar-Gomez E, Martinez-Perez Y, Calzadilla-Bertot $L$, et al Weight loss through lifestyle modification significantly reduces features of nonalcoholic steatohepatitis. Gastroenterology.
2015:149:367-78

31. Browning JD, Szczepaniak LS, Dobbins R, et al. Prevalence of hepatic steatosis in an urban population in the United States: impact of ethnicity. Hepatology. 2004:40:1387-95

32. Clark JM, Brancati FL, Diehl AM. Nonalcoholic fatty liver disease. Gastroenterology. 2002;122:1649-57.

33. Klair JS, Yang JD, Abdelmalek MF, et al. Nonalcoholic Steatohepatitis Clinical Research Network. A longer duration of estrogen deficiency increases fibrosis risk among postmenopausal women with nonalcoholic fatty liver disease Hepatology. 2016;64:85-91

34. Gambineri A, Pelusi V, Vicennati V, et al. Obesity and polycystic ovary disease. Int J Obesity. 2002;26:883-96

35. Kauffman RP, Baker TE, Baker V, et al. Endocrine factors associated with non-alcoholic fatty liver disease in women with polycystic ovary syndrome: do androgens play a role? polycystic ovary syndrome: do androge
Gynecol Endocrinol 2010:26:39-46.

36. Barbieri RL, Makris A, Randall RW, et al. Insulin stimulates androgen accumulation in incubations of ovarian stroma obtained from women with hyperandrogenism. J Clin Endocrinol Metab. 1986;62:904-10.

37. Kumarendran B, O'Reilly MW, Manolopoulos KN, et al. Polycystic ovary syndrome, androgen excess, and the risk of nonalcoholic fatty liver disease in women: a longitudinal study based on a United Kingdom primary care database. PLoS Med. 2018;15:e1002542.

38. Kupelian V, Page ST, Araujo AB, et al. Low sex hormone-binding globulin, total testosterone, and symptomatic androgen deficiency are associated with development of the metabolic syndrome in nonobese men. $J$ Clin Endocrinol Metab. 2006:91:843-50.

39. El baba K, Azar ST. Low testosterone and diabetes. Curr Diabetes Rev. 2013;9:418-21.

40. Couillard C, Gagnon J, Bergeron J, et al. C. Contribution of body fatness and adipose tissue distribution to the age variation in plasma steroid hormone concentrations in men: the HERITAGE Family Study. J Clin Endocrinol Metab. 2000;85:1026-31.

41. Kim S, Kwon H, Park J-H, et al. A low level serum total testosterone is independently associated with non-alcoholic fatty liver disease. BMC Gastroenterol. 2012;12:69.

42. Jaruvongvanich V, Sanguankeo A, Riangwiwat T, Upala S. Testosterone, sex hormone-binding globulin and nonalcoholic fatty liver disease: a systematic review and meta-analysis. Ann Hepatol. 2017;16:382-94.

43. Bhattacharya RK, Khera M, Blick G, et al. Effect of 12 months of testosterone replacement therapy on metabolic syndrome components in hypogonadal men: data from the Testim Registry in the US (TRiUS). BMC Endocr Disord. 2011:11:18

44. Hong JW, Kim JY, Kim YE, Lee EJ. Metabolic parameters and nonalcoholic fatty liver disease in hypopituitary men. 
Horm Metab Res. 2011;43:48-54

5. Koehler E, Swain J, Sanderson S, et al. Growth hormone, dehydroepiandrosterone and adiponectin levels in non-alcoholic steatohepatitis: an endocrine signature for .

46. Loomba R, Sirlin CB, Schwimmer JB, Lavine JE. Advances in pediatric nonalcoholic fatty liver disease. Hepatology. 2009;50:1282-93

47. Klieverik $\mathrm{LP}$, Coomans $\mathrm{CP}$, Endert $\mathrm{E}$, et al. Thyroid hormone effects on whole-body energy homeostasis and tissue-specific fatty acid uptake in vivo. Endocrinology. 2009;150;5639-48.

48. Pagadala MR, Zein CO, Dasarathy S, et al. Prevalence of hypothyroidism in nonalcoholic fatty liver disease. Dig Dis Sci. 2012;57:528-34.

49. Lomonaco R, Sunny NE, Bril F, Cusi K. Nonalcoholic fatty liver disease: current issues and novel treatment approaches. Drugs. 2013;73:1-14.

50. Glass LM, Dickson RC, Anderson JC, et al. Total body weight loss $\geq 10 \%$ is associated with improved fibrosis in patients with nonalcoholic steatohepatitis. Dig Dis Sci. 2015;60:1024-30.

51. Promrat K, Kleiner DE, Niemeier HM, et al. Randomized controlled trial testing the effects of weight loss on nonalcoholic steatohepatitis. Hepatology. 2010;51:121-9.

52. Uygun A, Kadayifci A, Sik AT, et al. Metformin in the treatment of patients with non-alcoholic steatohepatitis. Aliment Pharmaco Ther. 2004;19:537-44.

53. Cui J, Philo L, Nguyen P, et al. Sitagliptin vs placebo for non-alcoholic fatty liver disease: a randomized controlled trial. J Hepatol. 2016;65:369-76.

54. Takase T, Nakamura A, Miyoshi $\mathrm{H}$, et al. Amelioration of fatty liver index in patients with type 2 diabetes on ipraglifozlin: an association with glucose-lowering effects. Endocr $\rfloor$ 2017;64:363-7.

55. Ratziu V Giral P, Jacqueminet S, et al. Rosiglitazone for nonalcoholic steatohepatitis: one-year results of a randomized placebo controlled fatty liver improvement with rosiglitazone (FLIRT). Gastroenterology. 2008:135:100-10.

56. Musso G Cassader M. Paschetta E Gambino R.

Thiazolidinediones and advanced liver fibrosis in nonalcoholic steatohepatitis: a meta-analysis. JAMA Intern Med. 2017;177:633-40.

57. Lincoff AM, Wolski K, Nicholls SJ, Nissen SE. Pioglitazone and risk of cardiovascular events in patients with type 2 diabetes mellitus: a meta-analysis of randomized trials. JAMA. 2007;298:1180-8.

58. Younossi ZM, Loomba R, Rinella ME, et al. Current and future therapeutic regimens for nonalchoholic liver disease and non-alcoholic steatohepatitis. Hepatology 2018:68:361-71.

59. Townsend SA, Newsome PN. Review article: new treatments in non-alcoholic fatty liver disease. Aliment Pharmacol Ther. 2017;46:494-507.

60. Armstrong MJ, Gaunt P, Aithal GP, et al. Liraglutide safety and efficacy in patients with non-alcoholic steatohepatitis (LEAN): a multicentre, double-blind, randomised, placebo-controlled phase 2 study. Lancet. 2016;387:679-90

61. Armstrong MJ, Gaunt P, Aithal GP, et al. Safety and efficacy of liraglutide in patients with type 2 diabetes and elevated liver enzymes: individual patient data meta-analysis of the LEAD program. Aliment Pharmacol Ther. 2013:37:234-42.

62. Kaul U, Parmar D, Manjunath $\mathrm{K}$, et al. New dual peroxisome proliferator activated receptor agonist-Saroglitazar in diabetic dyslipidemia and non-alcoholic fatty liver disease: integrated analysis of the real world evidence. Cardiovasc Diabeto 2019;18:80.

63. Nelson A, Torres DM, Morgan AE, et al. A pilot study using simvastatin in the treatment of nonalcoholic steatohepatitis: a randomized placebo-controlled trial. J Clin Gastroenterol. 2009;43:990-4

64. Musso G, Gambino R, Cassader M, Pagano G. A meta-analysis of randomized trials for the treatment of nonalcoholic fatty liver disease. Hepatology. 2010;52:79-104

65. Lassailly G, Caiazzo R, Ntandja-Wandji L-C, et al. Bariatric surgery provides long-term resolution of nonalcoholic steatohepatitis and regression of fibrosis. Gastroenterology. 2020;159:1290-301.

66. Sjostrom $L$, Peltonen $M$, Jacobson $P$, et al. Bariatric surgery and long-term cardiovascular events. JAMA. 2012;307:56-65.

67. ClinicalTrials.gov. Randomized Global Phase 3 Study to Evaluate the Impact on NASH With Fibrosis of Obeticholic Acid Treatment (REGENERATE). ClinicalTrials.gov Identifier: NCT02548351. Available at: https://clinicaltrials.gov/ct2/show/NCT02548351 (accessed 31 March 2021)

68. ClinicalTrials.gov. Safety and Efficacy of Selonsertib in Adults With Compensated Cirrhosis Due to Nonalcoholic Steatohepatitis (NASH) (STELLAR-4). ClinicalTrials.gov Identifier: NCT03053063. Available at: https://clinicaltrials.gov/ct2/show/ NCT03053063 (accessed 31 March 2021)

69. ClinicalTrials gov Safety and Efficacy of Selonsertib in Adults With Nonalcoholic Steatohepatitis (NASH) and Bridging (F3) Fibrosis (STELLAR-3). ClinicalTrials gov Identifier: NCT03053050. Available at: https://clinicaltrials.gov/ct2/show/NCT03053050 accessed 31 March 2021).

70. ClinicalTrials.gov. Phase 3 Study to Evaluate the Efficacy and Safety of Elafibranor Versus Placebo in Patients With Nonalcoholic Steatohepatitis (NASH) (RESOLVE-IT). ClinicalTrials. gov Identifier: NCT02704403. Available at: https://clinicaltrials. gov/ct2/show/NCT02704403 (accessed 31 March 2021).

1. Armstrong MJ, Houlihan DD, Bentham L, et al. Presence and severity of non-alcoholic fatty liver disease in a large prospective primary care cohort. I Hepatol. 2012:56:234-40.

72. Bedogni G, Miglioli L, Masutti F, et al. Prevalence of and risk factors for non-alcoholic fatty liver disease. the Dionysos nutrition and liver study. Hepatology. 2005;42:44-52.

73. Angulo P, Hui JM, Marchesini G, et al. The NAFLD fibrosis score: a noninvasive system that identifies liver fibrosis in patients with NAFLD. Hepatology. 2007:45:846-54.

74. Clark JM, Brancati FL, DiehI AM. The prevalence and etiology of elevated aminotransferase levels in the United States
Am J Gastroenterol. 2003:98:960-7.

75. Castera L, Friedrich-Rust M, Loomba R. Noninvasive assessment of liver disease in patients with non-alcoholic fatty liver disease Gastroenterology. 2019:156:1264-81.e4.

76. Gao X, Fan J-G, Study Group of Liver and Metabolism, Chinese Society of Endocrinology. Diagnosis and management of non-alcoholic fatty liver disease and related metabolic disorders: consensus statement from the Study Group of Liver and Metabolism, Chinese Society of Endocrinology. J Diabetes. 2013;4:406-15

77. American Diabetes Association. Standards of medical care in diabetes - 2019 abridged for primary care providers. Clin Diabetes. 2019;37:11-34.

78. European Association for the Study of the Liver (EASL), European Association for the Study of Diabetes (EASD), and European Association for the Study of Obesity (EASO). EASL-EASD-EASO clinical practice guidelines for the management of non-alcoholic fatty liver disease. Obes Facts. 2016;9:65-90.

79. Petit JM, Cercueil J-P, Deimal D, et al. Effect of liraglutide therapy on liver fat content with inadequately controlled type-2 diabetes: the Lira-NAFLD study. J Clin Endocrinol Metab. 2017;102:407-15.

80. David JPE, Henry ZH, Argo CK, Northrup PG. Relationship of physician counseling to weight loss among patients with non-alcoholic liver disease: an observational cohort study using National Health and Education Survey data. Clin Liver Dis (Hoboken). 2019;14:156-60.

81. Targher G, Bertolini L, Rodella S, et al. NASH predicts plasma inflammatory biomarkers independently of visceral fat in men. Obesity. 2008;16:1394-9.

82. Shah AG, Lydecker A, Murray K, et al. Use of fib-4 index for non-invasive evaluation of fibrosis in non-alcoholic live disease Clin Gastroenterol Hepatol. 2009:7:1104-2.

83. Adams LA, George J, Bugiones E, et al. Complex non-invasive fibrosis models are more accurate than simple models in non-alcoholic fatty liver disease. $J$ Gastroenterol Hepatol. 2011;26:1536-43

84. Wong WW, Adams LA, de Ledinghen V, et al. Noninvasive biomarkers in NAFLD and NASH - current progress and future promise. Nat Rev Gastroenterol Hepatol. 2018;15:461-78.

85. Katsagoni CN, Georgoulis M, Papatheodoridis GV, et al. Effects of lifestyle interventions on clinical characteristics of patients with nonalcoholic fatty liver disease: a meta-analysis. Metabolism. 2017;68:119-32.

86. Koehler EM, Plompen EP, Schouten JN, et al. Presence of diabetes mellitus and steatosis is associated with liver stiffness in a general population: the Rotterdam study. Hepatology. 2016;63:138-47.

87. YounossizR, Stepanova M, Ong J, et al. Nonalcoholic steatohepatitis is the most rapidly increasing indication for liver transplantation in the United States. Clin Gastroenterol Hepatol. 2021:19:580-9. 